TRANSACTIONS OF THE

AMERICAN MATHEMATICAL SOCIETY

Volume 348, Number 5, May 1996

\title{
ON TRANSVERSELY FLAT CONFORMAL FOLIATIONS WITH GOOD MEASURES
}

\author{
TARO ASUKE
}

\begin{abstract}
Transversely flat conformal foliations with good transverse invariant measures are Riemannian in the $C^{1+\text { Lip }}$ sense. In particular, transversely similar foliations with good measures are transversely Riemannian as transversely $C^{\omega}$-foliations.
\end{abstract}

\section{INTRODUCTION}

The study of foliations with some transverse structure has attracted many people, and there are a lot of results for certain kinds of these foliations, such as Riemannian foliations, transversely affine foliations, and so on. In particular, transversely Riemannian foliations have been deeply studied, and we have the structure theorem (Molino [10]).

As a natural generalization, we can consider the transversely conformal foliations, which has been studied by Blumenthal [3], Vaisman [13], and others. This class is known to be quite large, so that all codimension one or transversely holomorphic foliations are transversely conformal.

Now we restrict our attention to foliations with transverse flat conformal structures. As is well-known, the flat conformal geometry appears as the geometry at infinity of the hyperbolic geometry.

It is well-known that certain kinds of flat conformal manifolds admit a Riemannian metric compatible with its flat conformal structure. This suggests that we may expect that, under some conditions, there exists a transverse invariant Riemannian metric for transversely flat conformal foliations, that is, the foliation is Riemannian.

In this paper we study transversely flat conformal foliations admitting nonatomic, full-support transverse invariant measures, which we call good measures, and obtain the following results.

Theorem A. Let $(M, \mathcal{F})$ be a transversely flat conformal foliation of a closed manifold $M$. Assume that there is a good measure $\mu$ on $M$. Then there is a transverse invariant Riemannian metric $g$ of $(M, \mathcal{F})$ which is of class $C^{1+\text { Lip }}$, namely, of class $C^{1}$ with the Lipschitz derivative.

The proof of Theorem A proceeds as follows. First, in section 3, we use slightly generalized versions of Ghys' lemmas [6] to show that the leaf space $\widehat{M}$ of the

Received by the editors May 8, 1995.

1991 Mathematics Subject Classification. Primary 53C12, 57R30, 53C10; Secondary 53A30, 57R20.

Key words and phrases. Foliation, transverse structure, invariant measure, Riemannian foliation, conformal structure.

(C)1996 American Mathematical Society 
universal covering of $M$ equipped with the lifted foliation of $\mathcal{F}$ is a Hausdorff manifold. Then in section 4 we apply the theorem of Kulkarni-Pinkall [8] which ensures the existence of a Riemannian metric of class $C^{1+\mathrm{Lip}}$ under the action of the full group $\operatorname{Conf}(\widehat{M})$ of conformal automorphisms as a Möbius manifold.

Remark. We do not know any example which satisfies all the assumptions of theorem A but is not a Riemannian foliation as a transversely $C^{\omega}$-foliation.

Now we assume that the foliation is of dimension one and all orbits are dense. For any flows on a closed manifold, there is a non-trivial transverse invariant ergodic measure $\mu$. The support of $\mu$ must be the whole manifold, and $\mu$ is of course non-atomic. Thus $\mu$ is good. So the theorem applies, and we obtain the following.

Corollary B. If $(M, \mathcal{F})$ is a transversely flat conformal flow of a closed manifold with dense orbits, then $(M, \mathcal{F})$ is transversely Riemannian in the $C^{1+\text { Lip }}$ sense.

In section 3 , we define the holonomy group of $(M, \mathcal{F})$. If the holonomy group is contained in $\operatorname{Sim}\left(\mathbf{R}^{q}\right)$, which is the case if the foliation is transversely similar, we have stronger results.

Theorem C. Let $(M, \mathcal{F})$ be a transversely flat conformal foliation of a closed manifold $M$ with a good measure. Assume that the holonomy group is contained in $\operatorname{Sim}\left(\mathbf{R}^{q}\right)$. Then $(M, \mathcal{F})$ is a Riemannian foliation as a transversely $C^{\omega}$ foliation.

In the paper [9] Matsumoto showed a similar result for flat conformal manifolds. To show Theorem C, we will generalize the method of Fried [5] and Matsumoto [9] to foliations under the existence of good measures. This step is done in section 5 .

In Carrière [4] he showed the following theorem.

Theorem. Let $(M, \mathcal{F})$ be a transversely Riemannian flow of a closed manifold with dense orbits. We assume that $M, \mathcal{F}$ are orientable and that $M, \mathcal{F}$, and the bundlelike metric on $M$ are of class $C^{r}, r \geq 2$. Then $(M, \mathcal{F})$ is differentiably conjugate to an irrational linear flow on the $n$-dimensional torus $T^{n}$. That is, there is a diffeomorphism from $M$ to $T^{n}$ which maps orbits to orbits.

Now let $(M, \mathcal{F})$ be a transversely flat conformal flow of a closed manifold with dense orbits. We assume that the holonomy group is contained in $\operatorname{Sim}\left(\mathbf{R}^{q}\right)$. Then by the same argument as in the proof of Corollary B, Theorem C applies in this case. Combining with the above theorem, we have the following.

Theorem D. Let $(M, \mathcal{F})$ be a transversely flat conformal flow of a closed manifold $M$ with all orbits dense. Suppose that $M, \mathcal{F}$ are of class $C^{r}, r \geq 2$, and orientable, and that the holonomy group is contained in $\operatorname{Sim}\left(\mathbf{R}^{q}\right)$. Then $(M, \mathcal{F})$ is $C^{r}$-conjugate to an irrational linear flow on the $n$-dimensional torus $T^{n}$ in the above sense.

In the paper [2], the author will classify the transversely flat conformal flows with good measures, which will show that under the hypothesis of Theorem $\mathrm{D}$, the flow is in fact an (Isom $\left.\left(\mathbf{R}^{q}\right), \mathbf{R}^{q}\right)$ flow. Notice that Theorem D is a complete analogue to the theorem of Carrière and that Theorems $\mathrm{C}$ and $\mathrm{D}$ are partial extention of the results by Nishimori [11] and Ghys [6].

Finally the author would like to express his gratitude to Professors T. Tsuboi and S. Matsumoto for their helpful suggestions and encouragement. 


\section{Definitions}

Throughout this paper, we work in the $C^{r}$-category, $r \geq 1$, unless otherwise stated. In the following, $\widehat{\mathbf{R}^{n}}\left(=\mathbf{R}^{n} \cup\{\infty\} \cong S^{n}\right)$ denotes the one point compactification of $\mathbf{R}^{n}$, and $\mathbf{R}^{+}$the positive real numbers.

Definition 1.1. We denote by $\operatorname{Sim}\left(\mathbf{R}^{q}\right)$ the group of similarity transformations of $\mathbf{R}^{q}$, namely, the group of transformations of $\mathbf{R}^{q}$ of the form $x \mapsto r \cdot A x+v$, where $r \in \mathbf{R}^{+}, A \in \mathrm{O}(q)$, and $v$ is a vector in $\mathbf{R}^{q}$, and we denote by $\operatorname{Conf}\left(\widehat{\mathbf{R}^{q}}\right)$ the group of Möbius transformations of $\widehat{\mathbf{R}^{q}}$, which is by definition the group generated by $\operatorname{Sim}\left(\mathbf{R}^{q}\right)$ and the inversion $J(x)=x /\|x\|^{2}$ with respect to the unit sphere. Notice that we can naturally consider $\operatorname{Sim}\left(\mathbf{R}^{q}\right) \subset \operatorname{Conf}\left(\widehat{\mathbf{R}^{q}}\right)$.

Notice that any conformal automorphism of $S^{q}$ is a Möbius transformation.

Let $M$ be an $n$-dimensional closed manifold and $\mathcal{F}$ a $q$-codimensional foliation of $M$. We say $(M, \mathcal{F})$ is transversely flat conformal if $(M, \mathcal{F})$ is a $\left(\operatorname{Conf}\left(S^{q}\right), S^{q}\right)$ foliation, namely, if there are an open covering $\left\{U_{i}\right\}_{i \in I}$ of $M$ and a family $\left\{f_{i}\right.$ : $U_{i} \rightarrow S^{q}$ of submersions such that

1) $\left\{\left(U_{i}, f_{i}\right)\right\}$ defines $\mathcal{F}$, i.e., $U_{i} \cong O_{i} \times T_{i}$ and $\left.\mathcal{F}\right|_{U_{i}}=\left\{O_{i} \times\{p\} ; p \in T_{i}\right\}$, where $O_{i}$ is an open set in $\mathbf{R}^{n-q}, T_{i}=f_{i}\left(U_{i}\right)$, and

2) the transition functions $\gamma_{j i}: f_{i}\left(U_{i} \cap U_{j}\right) \rightarrow f_{j}\left(U_{i} \cap U_{j}\right)$ are elements of $\operatorname{Conf}\left(S^{q}\right)$.

Here we may assume by decomposing the open set $U_{i}$ that $\overline{T_{i}} \neq S^{q}$, and that the index set $I$ is a finite set because $M$ is compact. Moreover we may assume that

3) Each $T_{i}$ is an open ball in $\mathbf{R}^{q}$.

So by changing $f_{i}$, we may assume

4) The closures $\overline{T_{i}}$ of $T_{i}$ are disjoint.

We say $(M, \mathcal{F})$ is transversely similar if $(M, \mathcal{F})$ is a $\left(\operatorname{Sim}\left(\mathbf{R}^{q}\right), \mathbf{R}^{q}\right)$ foliation.

Remark. Any transversely flat conformal foliation is transversely of class $C^{\omega}$ by definition.

We now put $T=\amalg T_{i}$, and $\Gamma$ denotes the pseudogroup associated with $T$. Since $M$ is compact, we can take concentric open balls $T_{i}^{\prime}$ in $T_{i}$ such that $\overline{T_{i}^{\prime}}$ is contained in $T_{i}$ and the pseudogroup $\Gamma^{\prime}$ associated with $T=\amalg T_{i}^{\prime}$ is equivalent to $\Gamma$.

By definition, $T$ and $T^{\prime}$ are contained in $\mathbf{R}^{q}$, and at the same time, we may regard them as submanifolds of $M$ in the natural way.

Definition 1.2. A transverse invariant measure $\mu$ of $(M, \mathcal{F})$ is said to be good if $\mu$ has the following properties.

a) $\operatorname{supp} \mu=M$, and

b) $\mu$ is non-atomic.

We also assume that $\mu$ is a Borel measure, and that $\mu$ is locally finite.

Notice that the required properties for measures are somehow natural, because every Riemannian foliation has such a measure even if we do not assume the differentiability.

Hereafter we always assume that $(M, \mathcal{F})$ has a good measure, and that $(M, \mathcal{F})$ is oriented and transversely oriented for simplicity. Thus when we consider transformation groups, we always assume that they consist of orientation preserving elements. 


\section{EXAMPLES}

Example 2.1. Let $M$ be a closed manifold of constant negative curvature and $\mathcal{F}$ the geodesic flow on the unit tangent bundle $U M$ of $M$. Then $(U M, \mathcal{F})$ has a dense orbit and a good measure but does not admit any transverse flat conformal structure. It is never Riemannian.

Example 2.2. Consider $S^{2}$, the unit sphere in $\mathbf{R}^{3}$, and define $\gamma_{1}$ and $\gamma_{2}$ as follows. First, let $B$ be the unit ball in $\mathbf{R}^{3}$ and put $C_{1}=B \cap\{y=z=0\}$ and $C_{2}=$ $B \cap\left\{x=0\right.$ and $\left.(y-\sqrt{2})^{2}+z^{2}=1\right\}$. Then we consider the Poincaré model on $B$ and define $\gamma_{1}$ and $\gamma_{2}$ to be the irrational rotations whose axes are $C_{1}$ and $C_{2}$, respectively.

Now let $\Sigma$ be a closed surface of genus two, $\Gamma$ the group generated by $\gamma_{1}$ and $\gamma_{2}$, and $(M, \mathcal{F})$ the suspension of $\left(S^{2}, \Gamma\right)$ over $\Sigma$. Then all leaves of $\mathcal{F}$ are dense and $(M, \mathcal{F})$ is transversely flat conformal but does not admit any good measure. It is easy to see that $(M, \mathcal{F})$ cannot be Riemannian.

This example shows that Corollary B cannot be generalized to higher dimensional foliations.

Example 2.3. Consider $\mathbf{R}^{2}=\mathbf{C}$ and the standard lattice $\mathbf{Z}^{2}$ in $\mathbf{C}$. We define $\gamma: \mathbf{C} \rightarrow \mathbf{C}$ by $\gamma(x, y)=(x+\theta, y+\varphi)$ and let $\Gamma$ be the group generated by $\gamma$. Here we consider $\mathbf{C} / \mathbf{Z}^{2}=T^{2}$, the 2 -dimensional torus.

Now we transport everything by the exponential map to $\mathbf{C} \backslash\{0\}$, and consider the suspension $(M, \mathcal{F})$ of $\left(T^{2}, \Gamma\right)$ over $S^{1}$. Then $(M, \mathcal{F})$ satisfies all the assumptions of Theorem $\mathrm{C}$, and is transversely Riemannian; in fact, $(M, \mathcal{F})$ is differentiably conjugate to a linear flow on the flat torus.

In particular, for suitable choices of $\theta$ and $\varphi$, all orbits of $\gamma$ are dense in $T^{2}$. Then Theorem D applies, and again we can see the foliation is transversely Riemannian as a transversely $C^{\omega}$ foliation.

Example 2.4. Consider the Hopf fibration $S^{3} \rightarrow S^{2}$ and let $X$ be a vector field tangent to the fibres. We choose $X$ appropriately so that if we consider its 1parameter group $\varphi_{t}$, then $\left\{\varphi_{n}(x)\right\}_{n \in \mathbf{Z}}$ is dense in the fibre passing through $x$.

Now we put $X=S^{3} \times(0, \infty)$, and define $\varphi, \psi$ as follows,

$$
\varphi(x, t)=\left(\varphi_{1}(x), r t\right) \quad \text { and } \quad \psi(x, t)=(x, 2 t) .
$$

Notice that if we consider $S^{3}$ as the unit sphere in $\mathbf{C}^{2}$, then we have

$$
\varphi_{1}\left(z_{1}, z_{2}\right)=\left(e^{2 \pi \sqrt{-1} \theta} z_{1}, e^{2 \pi \sqrt{-1} \theta} z_{2}\right),
$$

where $\theta$ is an irrational number. Therefore $\varphi$ is an orthogonal transformation.

Then we put $N=X /\langle\psi\rangle$ and consider the suspension $(M, \mathcal{F})$ of $(N, f)$, where $f$ is the automorphism induced by $\varphi$.

It is clear that $(M, \mathcal{F})$ is a $\left(\mathrm{CO}^{+}(4), \mathbf{R}^{4} \backslash\{0\}\right)$ flow, where $\mathrm{CO}^{+}(4)=\{h \in$ $\left.\operatorname{Sim}\left(\mathbf{R}^{4}\right) ; h(0)=0\right\}$. We equip $\mathbf{R}^{4} \backslash\{0\}$ with the metric defined by the formula

$$
g_{x}=\frac{1}{\|x\|^{2}} g_{E},
$$

where $g_{E}$ denotes the Euclidean metric and $\|\cdot\|$ denotes the Euclidean norm.

It is easy to see the action of $\mathrm{CO}^{+}(4)$ preserves this metric, thus $(M, \mathcal{F})$ is a Riemannian flow. 


\section{Preliminaries}

First of all, notice that since $T$ and $T^{\prime}$ are contained in $\mathbf{R}^{q}$, we have a natural Riemannian metric on $T$ and also on $T^{\prime}$ induced by the Euclidean metric on $\mathbf{R}^{q}$. We denote by $D_{\eta}(x)$ the open ball of radius $\eta$ and centered at $x$, and by $\operatorname{rad} D$ the radius of a ball $D$.

The following two lemmas appeared in Ghys [6].

Lemma 3.1. For any positive real number $\epsilon$, there is a positive real number $\eta$ with the following property:

If an element $\gamma \in \Gamma$ is defined on $D_{\eta}(x)$ and satisfies $\gamma x \in \overline{T^{\prime}}$, where $x \in T^{\prime}$, then $\operatorname{rad} \gamma D_{\eta}(x)$ is less than $\epsilon$.

Proof. First we claim that for any positive real number $\delta$, there is a positive real number $\eta$ such that $\mu\left(D_{\eta}\right)<\delta$ holds for any $D_{\eta} \subset T$ satisfying $D_{\eta} \cap \overline{T^{\prime}} \neq \phi$. For otherwise, we can find a positive real number $\delta$ and a sequence $\left\{B_{n}\right\}$ of open balls such that $\operatorname{rad} B_{n}=1 / n, B_{n} \cap \overline{T^{\prime}} \neq \phi$, and $\mu\left(B_{n}\right) \geq \delta$. Then we may assume $B_{n}$ converges to some point $y$ in $\overline{T^{\prime}}$, and $y$ is an atom of $\mu$. This contradicts the assumption that $\mu$ is non-atomic.

Secondly we show that for any positive real number $\epsilon$, there is a positive real number $\delta$ such that $\operatorname{rad} D<\epsilon$ holds for any open ball $D$ in $T$ satisfying $\mu(D)<\delta$ and $D \cap \overline{T^{\prime}} \neq \phi$. For otherwise, we can find a positive real number $\epsilon$ and a sequence $\left\{B_{n}\right\}$ of open balls satisfying $\mu\left(B_{n}\right)<1 / n, B_{n} \cap \overline{T^{\prime}} \neq \phi$, and $\operatorname{rad} B_{n}=\epsilon$. Now we may assume $\epsilon$ is less than the half of the distance between $\partial T$ and $\partial T^{\prime}$ in $\mathbf{R}^{q}$; then we may also assume that $B_{n}$ converges to some open ball $B$ in $T$. If we take an open ball $B^{\prime}$ such that $\overline{B^{\prime}} \subset B$, then we have $B^{\prime} \subset B_{n}$ for sufficiently large $n$. So we have $\mu\left(B^{\prime}\right) \leq \mu\left(B_{n}\right)<1 / n$, and hence $\mu\left(B^{\prime}\right)=0$ holds. This contradicts the assumption that $\mu$ is full-support.

Finally we show that for any positive real number $\epsilon$, there is a positive real number $\eta$ such that $\operatorname{rad} B_{1}<\eta$ implies $\operatorname{rad} B_{2}<\epsilon$ for any pair of open balls $B_{1}$ and $B_{2}$ in $T$ satisfying $B_{i} \cap \overline{T^{\prime}} \neq \phi, i=1,2$, and $\mu\left(B_{1}\right)=\mu\left(B_{2}\right)$. For the given positive real number $\epsilon$, choose $\delta$ as in the second claim and choose $\eta$ as in the first claim for this $\delta$. Then if $\operatorname{rad} B_{1}<\eta$, we have $\mu\left(B_{2}\right)=\mu\left(B_{1}\right)<\delta$ and consequently $\operatorname{rad} B_{2}<\epsilon$.

Now we prove Lemma 3.1. Since $\gamma$ maps balls to balls, preserves $\mu$, and $\gamma D_{\eta}(x) \cap$ $\overline{T^{\prime}} \ni \gamma x$, by putting $B_{1}=D_{\eta}(x)$ and $B_{2}=\gamma D_{\eta}(x)$, the above assertion applies and we complete the proof.

Lemma 3.2. There is a positive real number $\delta_{0}$ such that the germ $\gamma_{x}^{\prime}$ of an element of $\Gamma^{\prime}$ at any point $x$ in $T^{\prime}$ extends to an element of $\Gamma$ defined on $D_{\delta_{0}}(x)$.

Proof. Let $\Gamma_{1}^{\prime}$ denote the finite set of the natural generators of $\Gamma^{\prime}$, and $\Gamma_{k}^{\prime}$ denote the composition of $k$ elements of $\Gamma_{1}^{\prime}$. Then there is a positive real number $\delta$ such that for any $x$ in $T^{\prime}$ and the germ $\gamma_{x}^{\prime}$ of an element of $\Gamma_{1}^{\prime}$ at $x, \gamma_{x}^{\prime}$ extends to $\gamma \in \Gamma$ defined on $D_{\delta}(x)$. By Lemma 3.1, there is a positive real number $\delta_{0} \leq \delta$ such that $\operatorname{rad} \gamma D_{\delta_{0}}(x)<\frac{\delta}{2}$ holds for all $\gamma \in \Gamma$ defined on $D_{\delta_{0}}(x)$ with $\gamma x \in T^{\prime}$.

Now by the choice of $\delta_{0}$, Lemma 3.2 is true for $\Gamma_{1}^{\prime}$, so we assume that Lemma 3.2 is true for $\Gamma_{k}^{\prime}$ and prove it for $\Gamma_{k+1}^{\prime}$.

Let $\gamma \in \Gamma_{k+1}^{\prime}$ and write $\gamma=\gamma_{1} \gamma_{2}$, where $\gamma_{1} \in \Gamma_{1}^{\prime}$ and $\gamma_{2} \in \Gamma_{k}^{\prime}$. By the induction hypothesis, $\gamma_{2}$ is defined on $D_{\delta_{0}}(x)$ and $\gamma_{2} D_{\delta_{0}}(x) \subset D_{\delta}\left(\gamma_{2} x\right)$ holds. Since $\gamma_{1}$ is defined on $D_{\delta}\left(\gamma_{2} x\right), \gamma=\gamma_{1} \gamma_{2}$ is also defined on $D_{\delta_{0}}(x)$. 
Now we consider the universal covering $\widetilde{M}$ of $M$ and let $\widetilde{\mathcal{F}}$ be the lift of $\mathcal{F}$ to $\widetilde{M}$. Then we have the holonomy homomorphism $\phi: \pi_{1}(M) \rightarrow \operatorname{Conf}\left(S^{q}\right)$ and the developing map $D: \widetilde{M} \rightarrow S^{q}$. We denote by $H=\phi\left(\pi_{1}(M)\right)$ the holonomy group, and let $\widehat{M}=\widetilde{M} / \widetilde{\mathcal{F}}$. We denote by $p$ the natural projection from $\widetilde{M}$ to $\widehat{M}$.

Lemma 3.3. The leaf space $\widehat{M}$ is a Hausdorff manifold of dimension $q$.

Proof. It is well-known that $\widehat{M}$ is a possibly non Hausdorff manifold of dimension $q$. So it suffices to show that $\widehat{M}$ is Hausdorff. Suppose the contrary; then there are two points $x$ and $y$ of $\widetilde{M}$ belonging to distinct leaves of $\widetilde{\mathcal{F}}$, and two sequences $\left\{x_{n}\right\}$ and $\left\{y_{n}\right\}$ of $\widetilde{M}$ such that $x_{n}$ and $y_{n}$ belong to the same leaf for each $n$ and $x_{n}$ converges to $x$ and $y_{n}$ converges to $y$. We choose lifts $\widetilde{T}_{i}$ of $T_{i}$ and $\widetilde{T}_{i}^{\prime}$ of $T_{i}^{\prime}$ embedded in $\widetilde{M}$, respectively, and we may assume that $x, x_{n}$ are in $\widetilde{T}_{i}^{\prime}$ and $y, y_{n}$ are in $\widetilde{T}_{j}^{\prime}$ for some integers $i$ and $j$.

If we induce a metric to $\widetilde{T}_{i}^{\prime}$ from $T_{i}^{\prime}$ for each $i$, then clearly the conclusions of Lemmas 3.1 and 3.2 are true for the pseudogroups $\widetilde{\Gamma}$ and $\widetilde{\Gamma}^{\prime}$ of $\widetilde{\mathcal{F}}$ associated with $\widetilde{T}$ or $\widetilde{T}^{\prime}$.

Now we may assume $d\left(x_{n}, x\right)<\delta_{0}$ for all $n$; then any element $\widetilde{\gamma}$ of $\widetilde{\Gamma}^{\prime}$ which maps $x_{1}$ to $y_{1}$ is defined on $D_{\delta_{0}}\left(x_{1}\right)$, which contains $x$ and $x_{n}$ for any $n$. Noticing that the developing map $D$ is one to one on each $\widetilde{T}_{i}$ by definition, we see that $y_{n}$ is the only point which lies on the same leaf as $x_{n}$, or $\widetilde{\gamma}\left(x_{n}\right)$. Consequently we have $y_{n}=\widetilde{\gamma}\left(x_{n}\right)$ for each $n$, and hence $y=\widetilde{\gamma}(x)$. This is a contradiction, because we assumed that $x$ and $y$ are not in the same leaf.

\section{Proof of Theorem A}

We use the same notation as in the previous sections.

We have the action of $\pi_{1}(M)$ on $\widetilde{M}$ which preserves the lifted foliation $\widetilde{\mathcal{F}}$ of $\widetilde{M}$. So $\pi_{1}(M)$ naturally acts on $\widehat{M}$. On the other hand, the developing map $D$ obviously projects down to a mapping $\Delta: \widehat{M} \rightarrow S^{q}$, which is a local homeomorphism.

Now we have the action of $\pi_{1}(M)$ on $S^{q}$ via $\phi$. By definition of $\Delta$, it is clear that $\Delta$ is equivariant with respect to the action of $\pi_{1}(M)$, i.e., $\Delta(\gamma(x))=\phi(\gamma)(\Delta(x))$ holds for any $x \in \widehat{M}$ and $\gamma \in \pi_{1}(M)$.

If we find a Riemannian metric on $\widehat{M}$ which is invariant under the action of $\pi_{1}(M)$, then we have a transverse invariant metric on $\widetilde{M}$ which is invariant under the action of $\pi_{1}(M)$, and by projecting it down to $M$ we obtain a transverse invariant Riemannian metric on $M$. So our goal is to find an invariant metric on $\widehat{M}$.

We divide the study into the following three cases.

1) $\Delta$ is a homeomorphism onto $S^{q}$.

2) $\Delta$ is a homeomorphism onto $\mathbf{R}^{q}$.

3) Otherwise.

We will show in the rest of this section that $(M, \mathcal{F})$ is transversely Riemannian as a transversely $C^{\omega}$ foliation in cases 1 ) and 2), $C^{1+\text { Lip }}$ in case 3 ).

Remark. We will show in the next section that $(M, \mathcal{F})$ is a transversely $C^{\omega}$ Riemannian foliation if $H$ is contained in $\operatorname{Sim}\left(\mathbf{R}^{q}\right)$.

Notice that $\widehat{M}$ inherits a locally finite measure $\widehat{\mu}$ which is non-atomic and satisfies $\operatorname{supp} \widehat{\mu}=\widehat{M}$, and is invariant under the action of $\pi_{1}(M)$. 
First we treat case 1). We recall the notion of the limit sets of subgroups of $\operatorname{Conf}\left(S^{q}\right)$ and related facts. We consider $S^{q}=\widehat{\mathbf{R}^{q}}$ as the unit sphere in $\mathbf{R}^{q+1}$; then $\operatorname{Conf}\left(S^{q}\right)$ acts on the closed unit ball $D^{q+1}$ in $\mathbf{R}^{q+1}$ in the standard way.

Definition 4.1. We define the limit set $\Lambda(H)$ of $H$ in the same way as for the discrete groups, namely,

$$
\Lambda(H)=\left\{x \in S^{q} ; x=\lim _{n \rightarrow \infty} \gamma_{n}(p) \text { for some sequence }\left\{\gamma_{n}\right\} \text { of } H\right\},
$$

where $p$ is a point in $D^{q+1}$. It is easy to see that this definition is independent of the choice of the point $p$.

We say $H$ is elementary if $\Lambda$ consists of at most two points, non-elementary otherwise.

We use the following lemmas.

Lemma 4.2 ([9, Proposition 5.12]). For a subgroup $H$ of $\operatorname{Conf}\left(\widehat{\mathbf{R}^{q}}\right)$, the following conditions are equivalent.

1) $H$ is precompact.

2) $\Lambda(H)$ is empty.

Lemma 4.3 ([9, Proposition 5.13]). If a subgroup $H$ of $\operatorname{Conf}\left(S^{q}\right)$ is precompact, then $H$ is conjugate to a subgroup of $\mathrm{SO}(q+1) \subset \operatorname{Conf}\left(S^{q}\right)$ by an element of $\operatorname{Conf}\left(S^{q}\right)$.

We denote by $\mathcal{L}$ the closure of the set of fixed points of loxodromic elements of $H$. Recall that we say an element $h$ of $\operatorname{Conf}\left(S^{q}\right)$ is loxodromic if and only if $h$ has two fixed points in $S^{q}$.

The following proposition is well-known.

Proposition 4.4. If $\Lambda(H)$ consists of at least two points, then we have $\mathcal{L}=\Lambda(H)$.

Proof. See Theorem 5.15 and Lemma 5.6 of [9].

Now we have the following lemma.

Lemma 4.5. In case 1$), H$ is conjugate to a subgroup of $\mathrm{SO}(q+1)$.

Proof. In this case, we can define a finite measure $\bar{\mu}$ on $S^{q}$ by projecting down $\widehat{\mu}$. Then $\bar{\mu}$ is invariant under the action of $H$, non-atomic, and $\operatorname{supp} \mu=S^{q}$. Consequently, any element of $H$ is not loxodromic.

Now the above proposition shows that $\Lambda(H)$ consists of at most one point. So we assume that $\Lambda(H)$ consists of a single point. Then we may assume $\Lambda(H)=\{\infty\}$, and then $H$ is contained in $\operatorname{Sim}\left(R^{q}\right)$. Since $H$ preserves the good measure $\bar{\mu}$, any element of $H$ belongs to $\operatorname{Isom}\left(\mathbf{R}^{q}\right)$. Thus we can write any element $h$ of $H$ as

$$
h(x)=A_{h} x+v_{h} \text {, where } A_{h} \in \mathrm{SO}(q) \text {, and } v_{h} \in \mathbf{R}^{q} .
$$

Now we consider the upper half space model. So we regard $H$ as acting on $\overline{\mathbf{H}}^{q+1}=$ $\left\{x=\left(x_{1}, \ldots, x_{q+1}\right) \in \mathbf{R}^{q+1} ; x_{q} \geq 0\right\}$. We write an element $h$ of $H$ as $\widetilde{h}$ when we consider the action of $H$ on $\overline{\mathbf{H}}^{q+1}$. Then for $x=\left(x_{1}, \ldots, x_{q+1}\right) \in \overline{\mathbf{H}}^{q+1}$, we have

$$
\widetilde{h}\left(x_{1}, \ldots, x_{q+1}\right)=\left(A_{h} x^{\prime}+v_{h}, x_{q+1}\right), \text { where } x^{\prime}=\left(x_{1}, \ldots, x_{q}\right) .
$$


Since $\Lambda(H)=\{\infty\}$, we can find a sequence $\left\{h_{i}\right\}$ of elements of $H$ such that

$$
\lim _{i \rightarrow \infty} \widetilde{h}_{i}(0, \ldots, 0,1)=\infty
$$

holds. Then we have

$$
\lim _{i \rightarrow \infty} h_{i}\left(B_{1}\right)=\{\infty\},
$$

where $B_{1}$ denotes the open unit ball in $\mathbf{R}^{q}$. This implies $\infty$ is an atom of $\bar{\mu}$.

This is a contradiction, and we see that $\Lambda(H)$ is empty. Then Lemmas 4.2 and 4.3 prove the lemma.

Lemma 4.6. In case 2$), H$ is contained in $\operatorname{Isom}\left(\mathbf{R}^{q}\right)$.

Proof. As in the first case, we can project down $\widehat{\mu}$ to $\mathbf{R}^{q}$ and obtain a locally finite measure $\bar{\mu}$ on $\mathbf{R}^{q}$ which is invariant under the action of $H$.

Since every element of $H$ fixes the point $\infty$, we see that $H$ is a subgroup of $\operatorname{Sim}\left(\mathbf{R}^{q}\right)$. Now $\bar{\mu}$ is non-atomic, so $H$ is a subgroup of $\operatorname{Isom}\left(\mathbf{R}^{q}\right)$.

Lemmas 4.5 and 4.6 show that the foliation is transversely Riemannian as a transversely $C^{\omega}$ foliation in cases 1 ) and 2).

Now we assume case 3 ) holds and show that there is an invariant Riemannian metric on $\widehat{M}$ of class $C^{1+\text { Lip }}$. The only assumption we need is that $\widehat{M}$ is a Hausdorff manifold and $\Delta$ is neither a homeomorphism onto $S^{q}$ nor $\mathbf{R}^{q}$. In fact, we prove the following theorem.

Theorem 4.7. Let $(M, \mathcal{F})$ be a transversely flat conformal foliation of a closed manifold $M$. Assume that the leaf space of the lifted foliation $(\widetilde{M}, \widetilde{\mathcal{F}})$, where $\widetilde{M}$ denotes the universal covering, is a Hausdorff manifold. Assume also that the mapping $\Delta$ from $\widetilde{M}$ to $S^{q}$ induced by the developing map $D$ is a homeomorphism onto neither $S^{q}$ nor $\mathbf{R}^{q}$. Then there is a transverse invariant Riemannian metric $g$ of $(M, \mathcal{F})$ which is of class $C^{1+\text { Lip }}$. In particular the metric $g$ is twice differentiable almost everywhere with respect to the Lebesgue measure.

The above theorem is a direct consequence of the following.

Theorem 4.8 ([8, Theorem 5.9], see also [1]). Let $M$ be a Möbius manifold. Suppose that $M$ is neither a spherical space form nor a Euclidean space form. Then there is a complete Riemannian metric $g$ on $M$ compatible with the conformal structure of $M$ and of class $C^{1+\text { Lip }}$.

We say a Riemannian metric $g$ is compatible with the conformal structure if $g$ is invariant under the action of the full group of conformal automorphisms of $M$ as a Möbius manifold.

Notice that the Hopf-Rinow theorem holds for Riemannian metrics of class $C^{1+\text { Lip }}$, and we can speak of the completeness of such metrics.

Proof of Theorem 4.7. Let $\widehat{M}$ be as above. If $\widehat{M}$ is a spherical space form, we have the covering map

$$
\pi: S^{q} \rightarrow \widehat{M}
$$

The composition $\Delta \circ \pi$ gives a developing map from $S^{q}$ to $S^{q}$. The essential uniqueness of the developing map shows that $\pi$ is a diffeomorphism. The same argument 
shows that $\widehat{M}$ is diffeomorphic to $\mathbf{R}^{q}$ when $\widehat{M}$ is a Euclidean space form. Therefore $\widehat{M}$ is neither a spherical space form nor a Euclidean space form.

Then Theorem 4.8 shows that there is a Riemannian metric $g$ of $\widehat{M}$ which is of class $C^{1+L i p}$ and invariant under the action of the full group of conformal transformations of $\widehat{M}$ as a Möbius manifold. Now it is clear that the natural action of $\pi_{1}(M)$ on $\widehat{M}$ preserves the conformal structure of $\widehat{M}$.

Thus the metric $g$ is invariant under the action of $\pi_{1}(M)$, and we complete the proof.

We have a good description of the mapping $p: \widetilde{M} \rightarrow \widehat{M}$.

Proposition 4.9. The mapping $p: \widetilde{M} \rightarrow \widehat{M}$ is a locally trivial fibration.

Proof. First we fix a smooth Riemannian metric $g_{0}$ on $M$ and let $\widehat{g_{T}}$ denote the invariant metric on $\widehat{M}$ given in the proof of Theorem 4.7. Then the lift $\widetilde{g}_{0}$ of $g_{0}$ to $\widetilde{M}$ defines an orthogonal distribution $\widetilde{P}$ of $T \widetilde{\mathcal{F}}$.

Noticing that $p_{*}$ gives an isomorphism from $\widetilde{P}$ to $T \widehat{M}$, we have a $C^{1+\text { Lip }}$ Riemannian metric $\widetilde{g}_{T}$ on $\widetilde{P}$ which projects down to the metric $\widehat{g_{T}}$ on $\widehat{M}$. We denote by $\|\cdot\|_{\widetilde{g}_{T}}$ the norm on $\widetilde{P}$ induced by $\widetilde{g}_{T}$ and by $\|\cdot\|_{\widehat{g_{T}}}$ the norm on $\widehat{M}$ induced by $\widehat{g_{T}}$.

We define $\widetilde{g}_{\widetilde{\mathcal{F}}}$ to be the restriction of $\widetilde{g}_{0}$ to $T \widetilde{\mathcal{F}}$ and put $\widetilde{g}=\widetilde{g}_{\widetilde{\mathcal{F}}} \oplus \widetilde{g}_{T}$; then $\widetilde{g}$ projects down to a bundle-like metric $g$ on $M$ with respect to $\mathcal{F}$. The metric $g$ is of class $C^{1+\text { Lip }}$ and complete, hence $\widehat{g}$ is also.

Now we fix a point $x_{0}$ in $\widehat{M}$ and choose an open neighbourhood $V$ of $x_{0}$ such that there is a positive real number $R$ such that $d(p, q)<R$ holds for any points $p$ and $q$ in $V$, where $d$ is the distance function on $\widehat{M}$ determined by $\widehat{g_{T}}$.

Let $x$ and $y$ be points in $V$. We consider the geodesic $\sigma_{x, y}:[0,1] \rightarrow V$ with respect to $\widehat{g_{T}}$ joining $x$ and $y$. Now we fix a point $\widetilde{x}$ in $p^{-1}(x)$ and consider the horizontal lift $\sigma_{x, y}(\widetilde{x})$ of $\sigma_{x, y}$ starting at $\widetilde{x}$. It is clear that such a lift is unique if it exists. So we show the existence.

Since we can always find the lift locally, first we assume that $\sigma_{x, y}(\widetilde{x})$ is defined on $[0, s), s \leq 1$. We then have that

$$
\begin{aligned}
\int_{0}^{s}\left\|\frac{d \sigma_{x, y}(\widetilde{x})}{d t}\right\|_{\widetilde{g}} d t & =\int_{0}^{s}\left\|\frac{d \sigma_{x, y}(\widetilde{x})}{d t}\right\|_{\widetilde{g}_{T}} d t \\
& =\int_{0}^{s}\left\|\frac{d \sigma_{x, y}}{d t}\right\|_{\widehat{g_{T}}} d t \\
& \leq \int_{0}^{1}\left\|\frac{d \sigma_{x, y}}{d t}\right\|_{\widehat{g_{T}}} d t \\
& \leq R .
\end{aligned}
$$

Hence the image of $\sigma_{x, y}(\widetilde{x})$ is contained in $K$, where $K$ is the closed ball centred at $x_{0}$ and of radius $R$ with respect to the metric $\widehat{g}$. Now the completeness of $\widehat{g}$ implies that $K$ is compact.

Thus any horizontal lift $\sigma_{x, y}(\widetilde{x})$ of $\sigma_{x, y}$ stays in a fixed compact set $K$, and the

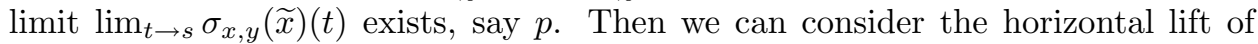
$\sigma_{x, y}$ near $p$, which must coincide with $\sigma_{x, y}(\widetilde{x})$ near $p$. So we can extend $\sigma_{x, y}(\widetilde{x})$ to 
$[0, s+\epsilon)$ for some positive real number $\epsilon$. This lift also stays in $K$, and hence we can extend $\sigma_{x, y}(\widetilde{x})$ to $[0,1]$.

Finally notice that $\sigma_{x, y}(\widetilde{x})$ is a geodesic with respect to $\widehat{g}$, and we define a mapping $\phi: p^{-1}(x) \times V \rightarrow p^{-1}(V) \subset \widetilde{M}$ by

$$
\phi(\widetilde{x}, y)=\sigma_{x, y}(\widetilde{x})(1) ;
$$

then $\phi$ is a continuous map. We show that $\phi$ is a homeomorphism. In fact, for $\widetilde{z} \in p^{-1}(V)$ we put $z=p(\widetilde{z})$ and consider $\sigma_{z, x}$. Then $\sigma_{z, x}$ lifts to a path $\sigma_{z, x}(\widetilde{z})$. We put $\widetilde{x}_{z}=\sigma_{z, x}(\widetilde{z})(1)$ and then obtain a mapping $\Psi: p^{-1}(V) \rightarrow p^{-1}(x) \times V$ such that $\Psi(\widetilde{z})=\left(\widetilde{x}_{z}, z\right)$. Clearly $\Psi$ is continuous.

Now consider the path $\sigma_{x, z}\left(\widetilde{x}_{z}\right)$. The uniqueness of the lift implies that this path coincides with the path $t \mapsto \sigma_{z, x}(\widetilde{z})(1-t)$. Hence $\phi \circ \Psi$ is the identity map.

On the other hand, we suppose that $\widetilde{z}=\phi(\widetilde{x}, y)=\phi\left(\widetilde{x}^{\prime}, y^{\prime}\right)$. This is equivalent to saying $\widetilde{z}=\sigma_{x, y}(\widetilde{x})(1)=\sigma_{x, y^{\prime}}\left(\widetilde{x}^{\prime}\right)(1)$. Since the definition of $\phi$ shows that we have $p \circ \phi(\widetilde{x}, y)=y$, we have $y=y^{\prime}$. Now we reverse the direction of both paths $\sigma_{x, y}(\widetilde{x})$ and $\sigma_{x, y}\left(\widetilde{x}^{\prime}\right)$. Then they are the horizontal lifts of $\sigma_{y, x}$ starting at $\widetilde{z}$. Again the uniqueness shows that these paths coincide, in particular $\widetilde{x}=\widetilde{x}^{\prime}$. Thus $\Psi \circ \phi$ is the identity.

Consequently $\Psi$ gives a local triviality.

As a corollary we have the following, which is shown for complete transversely conformal foliations in Blumenthal [3].

Corollary 4.10. The leaves of $(M, \mathcal{F})$ have the same universal covering.

In the paper [7] Heitsch and Hurder conjectured that if $(M, \mathcal{F})$ admits a full support measure. Then all the Godbillon-Vey classes [7] are zero. Related to this conjecture, we have the following corollary.

Corollary 4.11. Let $(M, \mathcal{F})$ be a transversely flat conformal foliation of a closed manifold. Suppose that $(M, \mathcal{F})$ admits a good measure, then all the Godbillon-Vey classes of $(M, \mathcal{F})$ vanish.

Proof. Since $(M, \mathcal{F})$ is continuously Riemannian, there is a continuous distance function on $T$ which is invariant under the action of the holonomy pseudogroup. Then Theorem 4.3 of [7] shows that all the Godbillon-Vey classes of $(M, \mathcal{F})$ are zero.

One can ask how the regularity of the metric can be higher as the regularity of the good measure $\mu$ becomes higher. On this line, we have the following.

Recall that a good measure $\mu$ defines a $\Gamma$-invariant measure, denoted by $\mu_{T}$, on $T$. For an integer $r \geq 0$, a good measure $\mu$ is called a $C^{r}$-volume form if we have $\mu_{T}=f \cdot \lambda$, where $\lambda$ denotes the Lebesgue measure restricted to $T$ and $f$ is a positive $C^{r}$-function on $T$.

Proposition 4.12. Let $(M, \mathcal{F})$ be a transversely flat conformal foliation preserving $\mu$. Let $\mu$ be a $C^{r}$-volume form. Then we can find an invariant metric $g_{\mu}$ of class $C^{r}$.

Proof. First notice that we have

$$
f \circ \gamma(x) \cdot \operatorname{det} \gamma_{*}=f(x)
$$


for any $\gamma \in \Gamma$ and $x$ in the domain of $\gamma$. This is because $f$ is continuous and the above equation holds a.e. by virtue of the equation $\gamma^{*} \mu=\mu$.

Now we denote by $g^{E}$ the Euclidean metric restricted to $T$ and define

$$
g_{x}=f(x)^{\frac{2}{q}} g^{E},
$$

where $q$ is the codimension of $\mathcal{F}$.

Notice that for an orientation preserving element $\gamma$ of $\operatorname{Conf}\left(\mathbf{R}^{q}\right), \gamma_{*}$ is an element of $\mathrm{SO}(q)$ and we have

$$
\left(\gamma^{*} g^{E}\right)_{\gamma x}=\left(\operatorname{det} \gamma_{* x}\right)^{\frac{2}{q}} g_{x}^{E}
$$

Then we have

$$
\begin{aligned}
\left(\gamma^{*} g\right)_{\gamma x} & =f(\gamma(x))^{\frac{2}{q}}\left(\gamma^{*} g^{E}\right)_{\gamma x} \\
& =f(\gamma(x))^{\frac{2}{q}}\left(\operatorname{det} \gamma_{* x}\right)^{\frac{2}{q}} g^{E}{ }_{x} \\
& =f(x)^{\frac{2}{q}} g^{E}{ }_{x} \\
& =g_{x} .
\end{aligned}
$$

Thus $g$ is invariant under the action of $\Gamma$.

In general the invariant metric $g_{T}$ in Theorem 4.7 and $g_{\mu}$ in Proposition 4.12 need not be the same. However, if there is a dense leaf, then $g_{T}$ and $g_{\mu}$ are essentially the same.

Lemma 4.13. If $(M, \mathcal{F})$ satisfies the conditions Proposition 4.12, and admits a dense leaf, then there is a positive real number c such that $g_{T}=c \cdot g_{\mu}$.

Proof. We regard the metrics $g_{T}$ and $g_{\mu}$ as the metrics on $T$ invariant under the action of $\Gamma$. Since there is a dense leaf, we can find a point $x_{0}$ in $T$ such that $\Gamma x_{0}$ is dense in $T$.

Let $c$ be the positive real number $c$ such that

$$
\left(g_{T}\right)_{x_{0}}=c \cdot\left(g_{\mu}\right)_{x_{0}} .
$$

Then the invariance of the metrics implies that

$$
\left(g_{T}\right)_{x}=c \cdot\left(g_{\mu}\right)_{x}
$$

for $x \in \Gamma x_{0}$. Since both sides are continuous and $\Gamma x_{0}$ is dense in $T$, the above equation holds on $T$.

\section{The transversely similar CASE}

In this section we deal with the case where the holonomy group $H$ is contained in $\operatorname{Sim}\left(\mathbf{R}^{q}\right)$. This is the case when the foliation is transversely similar. We prove Theorem $\mathrm{C}$ in this section.

The case where the foliation is transversely similar and of codimension two has already been treated in [6] without the assumption on measures, and there is nothing to prove. But our assumption is slightly weaker than that of [6]. So we assume 
the codimension of the foliation is greater than one, and the foliation $\mathcal{F}$ has a good measure $\mu$. We retain the same notation as in the previous sections.

Notice that that if $\Delta$ is a homeomorphism onto $S^{q}$ or $\mathbf{R}^{q}$, then we have already shown the above theorem. So we assume that $\Delta$ is a homeomorphism onto neither $S^{q}$ nor $\mathbf{R}^{q}$.

The proof will proceed almost in the same way as in [9] or Fried [5]. But we follow it again since it is the heart of the proof of Theorem $\mathrm{C}$ and the very base of the classification of flows in [2].

Definition 5.1. We define

$$
\begin{aligned}
\widehat{M}(\infty) & =\Delta^{-1}(\infty), \\
M(\infty) & =\pi\left(D^{-1}(\infty)\right)=\pi\left(p^{-1} \Delta^{-1}(\infty)\right), \\
\widehat{M}^{*} & =\widehat{M} \backslash \widehat{M}(\infty), \text { and } \\
M^{*} & =M \backslash M(\infty)=\pi\left(\widetilde{M} \backslash D^{-1}(\infty)\right) .
\end{aligned}
$$

Lemma 5.2. $M(\infty)$ is a union of a finite number of compact leaves.

Proof. Suppose the contrary. Then there is a point $p$ of $M$ where the leaves $\left\{L_{i}\right\}$ of $M(\infty)$ accumulate. (The set $\left\{L_{i}\right\}$ may consist of a single non-compact leaf.) This shows that if we choose any lift $\left\{\widetilde{L}_{i}\right\}$ of $\left\{L_{i}\right\}$, then $\Omega$, the orbit of $\left\{p\left(\widetilde{L}_{i}\right)\right\}$ by $\pi_{1}(M)$, has an accumulating point. Since any element of $H$ fixes infinity, $\Omega$ is contained in $\widehat{M}(\infty)$. On the other hand, $\widehat{M}(\infty)$ is discrete because $\Delta$ is a local homeomorphism. This yields a contradiction.

We write $M(\infty)=\bigcup_{i=1}^{l} L_{i}$, where each $L_{i}$ is a compact leaf.

Definition 5.3. A domain $\widehat{U}^{*}$ of $\widehat{M}^{*}$ is called a copy of a domain $U$ of $\mathbf{R}^{q}$ if the restriction of $\Delta$ to $\widehat{U}^{*}$ is a homeomorphism.

Definition 5.4. We say that a closed subset $\widehat{l}^{*}$ of $\widehat{M}^{*}$ is a complete half line if for any copy $\widehat{B}^{*}$ of an open ball $B$ in $\widehat{M}^{*}$, the image of $\widehat{B}^{*} \cap \widehat{l}^{*}$ by $\Delta$ is equal to $B \cap l$, where $l$ is a complete half line in $\mathbf{R}^{q}$.

A complete half line $\widehat{l}^{*}$ is called short if $l=\Delta\left(\widehat{l}^{*}\right)$ is not a complete half line in $\mathbf{R}^{q}$. We always parametrize $l$ and $\widehat{l}^{*}$ as follows.

Let $\alpha$ and $\beta$ be the endpoints of $l$ such that $\alpha \in l$ and $\beta \notin l$. Then we define

$$
l(t)=\frac{1}{t+1}(\alpha+t \beta)
$$

for $t \in[0, \infty)$, and parametrize $\widehat{l}^{*}$ so that $\Delta(\widehat{l} *(t))=l(t)$ holds.

Lemma 5.5. For any short complete half line $\widehat{l}^{*}$, there is a compact saturated neighbourhood $U$ of $M(\infty)$ which is disjoint from $\pi\left(p^{-1}\left(\widehat{l}^{*}\right)\right)$.

Proof. For any leaf $L_{i}$ of $M(\infty)$, there is a compact saturated neighbourhood $U_{i}$ of $L_{i}$ which is disjoint from $\pi\left(p^{-1} \widehat{l}^{*}(0)\right)$. For, if not, then $\pi\left(p^{-1} \widehat{l}^{*}(0)\right)$ must accumulate to $L_{i}$. In other words, $L_{i}$ has a contracting holonomy. This is a contradiction because we have already shown that $(M, \mathcal{F})$ is continuously Riemannian. 
Now by shrinking $U_{i}$, we may assume that $U_{i}$ is evenly covered by $\pi$, and for each connected component $\widehat{E}_{k}^{*}$ of $p\left(\pi^{-1}\left(U_{i}\right)\right), \Delta$ is a homeomorphism from $\widehat{E}_{k}^{*}$ onto $E_{k}=E\left(a_{k}, R_{k}\right)=\left\{\left|x-a_{k}\right| \geq R_{k}\right\} \cup\{\infty\}$.

Then we have $\widehat{l}^{*} \cap \widehat{E}_{k}^{*}=\phi$. The reason is as follows. Suppose the contrary then $l \cap E_{k}$ is a half line starting at a point of $\partial E_{k}$, where $l=\Delta\left(\widehat{l}^{*}\right)$. Since $\widehat{l}^{*}$ is short, $p=\lim _{t \rightarrow \infty} l(t)$ exists. But for any copy $\widehat{B}^{*}$ of an open ball $B$ centered at $p$, its intersection with $\widehat{l}^{*}$ cannot be mapped to $B \cap l$ by $\Delta$. This contradicts the completeness of $\widehat{l}^{*}$.

So $p^{-1}\left(\widehat{l}^{*}\right) \cap p^{-1}\left(\widehat{E}_{k}^{*}\right)=\phi$ for each component $\widehat{E}_{k}^{*}$, and consequently we have $\pi\left(p^{-1}\left(\widehat{l}^{*}\right)\right) \cap U_{i}=\phi$. Now we define $U=\bigcup_{i=1}^{l} U_{i}$; then $\pi\left(p^{-1}\left(\hat{l}^{*}\right)\right) \cap U=\phi$.

Definition 5.6. For a point $\widehat{x} \in \widehat{M}^{*}$, we define $r(\widehat{x})$ to be the maximum of the radii of copies of balls centered at $\widehat{x}$.

From now on we will denote by $x$ the image of a point $\widehat{x}$ of $\widehat{M}^{*}$ under $\Delta$.

Lemma 5.7. The function $r$ is of finite value.

Proof. Suppose $r(\widehat{x})=\infty$; then $\widehat{x}$ is contained in a copy $\widehat{P}$ of $\mathbf{R}^{q}$. Since we assumed that $\Delta$ is not a homeomorphism, we have $\widehat{M}^{*} \neq \widehat{P}$. So we can find a point $\widehat{y}$ of $\partial \widehat{P}$ and a sequence $\left\{\widehat{y_{n}}\right\}$ of $\widehat{P}$ converging to $y$.

Since $\Delta\left(\widehat{y_{n}}\right)$ tends to $\infty$, we have $D(\widehat{y})=\infty$. Therefore there is a neighbourhood $Q$ of $\widehat{y}$ which is mapped to $E(0, R)$ by $\Delta$ for some positive real number $R$. Then $\Delta$ is a homeomorphism from $P \cup Q$ to $S^{q}$. Since $P \cup Q$ is open and closed in $\widehat{M}$, we see that $\widehat{M}=P \cup Q$. This contradicts our assumption.

Lemma 5.8. The function $r$ is continuous.

Proof. Let $\widehat{x}$ be a point in $\widehat{M}$ and let $\widehat{B}$ be the open ball centred at $\widehat{x}$, of radius $r(\widehat{x})$. We consider the Euclidean metric on $B=\Delta(\widehat{B})$.

Let $y$ be a point in $B$ such that the distance between $x$ and $y$ is less than $\epsilon$. Then the open ball centred at $y$ and of radius $r(\widehat{x})-\epsilon$ clearly has a copy, so $r(\widehat{y})>r(\widehat{x})-\epsilon$. Conversely, the same argument shows that $r(\widehat{y})>r(\widehat{x})-\epsilon$.

Definition 5.9. We define a continuous Riemannian metric $\widehat{g}$ on $\widehat{M}^{*}$ by

$$
\widehat{g}_{\widehat{x}}=\frac{1}{r(\widehat{x})^{2}}\left(\Delta^{*} g_{0}\right) \widehat{x}
$$

where $g_{0}$ denotes the Euclidean metric on $\mathbf{R}^{q}$.

Remark 1. $\widehat{g}$ is, in a manner of speaking, the transverse Fried metric.

Remark 2. This definition is an analogue of Definition 5.2 of [8]. In the present case the centres of balls are preserved under the action of $\pi_{1}(M)$ or $H$, so we can consider normal maximal balls centred at $\hat{x}$. By using this notion instead of normal maximal balls, we obtain this metric.

The following lemma is clear from the definition.

Lemma 5.10. $\widehat{g}$ is invariant under the action of $\pi_{1}(M)$.

Hence we have a transverse invariant metric $g$ on $M^{*}$. 
We define a function $\widehat{d}=\widehat{d_{g}}: \widehat{M}^{*} \times \widehat{M}^{*} \rightarrow \mathbf{R}$ by the formula

$$
\widehat{d}(p, q)=\inf _{\rho} \int_{0}^{1}\left\|\frac{d \rho}{d t}(t)\right\|_{\widehat{g}} d t
$$

where $\rho:[0,1] \rightarrow \widehat{M}^{*}$ runs through piecewise smooth paths in $\widehat{M}^{*}$ such that $\rho(0)=p$ and $\rho(1)=q$.

It is easy to see that $\widehat{d}$ is of finite value on $\widehat{M}^{*} \times \widehat{M}^{*}$, and is continuous. Since we have $\widehat{d}(\gamma p, \gamma q)=\widehat{d}(p, q)$, we have a continuous function $d$ on $M^{*} \times M^{*}$ which locally coincides with $\widehat{d}$.

Hereafter we will describe $\widehat{g}$ more explicitly and make it clear that $\widehat{g}$ is of class $C^{\omega}$.

Proposition 5.11. Let $\widehat{B}=\widehat{D}_{r(\widehat{a})}(\widehat{a})$ be the maximal copy of an open ball. Then there is a unique copy of a half space $\widehat{H}$ which contains $\widehat{B}$.

Proof. First we show the existence. Without loss of generality, we may assume that $r(\widehat{a})=1$ and $\Delta(\widehat{a})=e=(0, \ldots, 0,1)$. Since $\Delta$ restricted to $\widehat{B}$ is a homeomorphism, we may assume

$$
\left.\widehat{g}\right|_{\widehat{B}}=\frac{1}{r(\widehat{x})^{2}}\left(g_{0}\right)_{\widehat{x}}
$$

where $\widehat{B}$ is identified with $B$.

Now by the maximality of $\widehat{B}$, we can find a radius $\widehat{l}^{*}$ of $\widehat{B}$ which is a complete half line. We may assume $l=\Delta\left(\widehat{l}^{*}\right)=\left\{x_{1}=\cdots=x_{q-1}=0,0<x_{q} \leq 1\right\}$. Then the parametrization of $l$ is

$$
l(t)=\left(0, \ldots, 0, \frac{1}{t+1}\right)
$$

where $t \in[0, \infty)$.

Let $\widehat{x}$ be a point in $\widehat{B}$. We define

$$
\theta(\widehat{x})=\arccos \frac{\left|g_{0 o}(\overrightarrow{o x}, \overrightarrow{o e})\right|}{\|x\| \cdot\|e\|}
$$

to be the angle of $x=\Delta(\widehat{x})$ and $e$ considered as vectors, where $o$ is the origin of $\mathbf{R}^{q}$. Then we have following sublemmas.

Sublemma 5.12. We have

$$
\widehat{d}\left(\widehat{x}, \widehat{l_{q}}\right) \geq \theta(x), \quad \text { for } \widehat{x} \in \widehat{B},
$$

where $l_{q}=\left\{x_{1}=\cdots=x_{q-1}=0,0<x_{q}<2\right\} \subset B$ and $\widehat{l_{q}}$ is the lift of $l_{q}$ to $\widehat{B}$.

Proof of Sublemma 5.12. We work in $B$, and it suffices to show that

$$
\bar{d}\left(x, l_{q}\right) \geq \theta(x),
$$

where $\bar{d}$ is the metric obtained from $\widehat{d}$ by projecting by the homeomorphism $\left.\Delta\right|_{\widehat{B}}$. 
Let $\gamma$ be a piecewise smooth path in $B$ from $x$ to a point in $l_{q}$. So $\gamma$ is a mapping from $[0,1]$ to $B$.

We denote by $\|\cdot\|$ the Euclidean metric. We write $\gamma(t)=\|\gamma(t)\| \varphi(t)$; then we have

$$
\frac{d \gamma}{d t}(t)=\frac{d\|\gamma\|}{d t}(t) \varphi(t)+\|\gamma(t)\| \frac{d \varphi}{d t}(t) .
$$

On the other hand, since $\|\varphi(t)\| \equiv 1$, we have $g_{0}\left(\varphi(t), \frac{d \varphi}{d t}(t)\right) \equiv 0$. So we have

$$
\begin{aligned}
\left\|\frac{d \gamma}{d t}(t)\right\|^{2} & =\left\|\frac{d\|\gamma\|}{d t}(t)\right\|^{2}\|\varphi(t)\|^{2}+\|\gamma(t)\|^{2}\left\|\frac{d \varphi}{d t}(t)\right\|^{2} \\
& \geq\|\gamma(t)\|^{2}\left\|\frac{d \varphi}{d t}(t)\right\|^{2} .
\end{aligned}
$$

Now we define

$$
\operatorname{length}_{\bar{d}}(\gamma)=\int_{0}^{1}\left\|\frac{d \gamma}{d t}(t)\right\|_{\bar{g}} d t
$$

where $\bar{g}$ denotes the metric obtained from $\widehat{g}$ by projecting by $\left.\Delta\right|_{\widehat{B}}$.

We claim that $r(\widehat{y}) \leq\|y\|$ holds for any $\widehat{y} \in \widehat{B}$. Suppose the contrary; then the origin of $\mathbf{R}^{q}$ is contained in $A=B \cup\left\{x \in \mathbf{R}^{q} ;\|x-y\|<r(\widehat{y})\right\}$, which has a copy containing $\widehat{a}$. This contradicts the completeness of $\widehat{l}^{*}$, since the projected image of the intersection of $l$ and any copy of an open ball $B_{0} \subset A$ centred at the origin is not of the form $l^{\prime} \cap B_{0}$, where $l^{\prime}$ is a complete half line in $\mathbf{R}^{q}$. Consequently we have

$$
\widehat{g}_{\widehat{x}} \geq \frac{1}{\|x\|^{2}} g_{0} .
$$

It follows that we have

$$
\begin{aligned}
\operatorname{length}_{\bar{d}}(\gamma) & \geq \int_{0}^{1} \frac{\left\|\frac{d \gamma}{d t}(t)\right\|}{\|\gamma(t)\|} d t \\
& \geq \int_{0}^{1}\left\|\frac{d \varphi}{d t}(t)\right\| d t \\
& \geq \theta(x) .
\end{aligned}
$$

This implies $\bar{d}\left(x, l_{q}\right) \geq \theta(x)$.

Sublemma 5.13. If $\widehat{y} \in \widehat{M}^{*}$ is not contained in $\widehat{B}$, then there is a positive real number $\epsilon_{0}$ such that $\widehat{d}\left(\widehat{y}, \widehat{l}^{*}\right)>\epsilon_{0}$ holds.

Proof of Sublemma 5.13. We identify $\widehat{B}$ and $B$, and define

$$
\begin{aligned}
& C_{\varphi}=\{\widehat{x} \in \widehat{B} ; \theta(\widehat{x})=\varphi\}, \\
& B_{l}=\left\{\widehat{x}=\left(x_{1}, \ldots, x_{q}\right) \in \widehat{B} ;\|\overrightarrow{e \widehat{x}}\|=\frac{4}{3}, x_{q}>1\right\}, \\
& B_{m}=\left\{\widehat{x}=\left(x_{1}, \ldots, x_{q}\right) \in \widehat{B} ; \frac{4}{3} \leq\|\overrightarrow{e \widehat{x}}\| \leq \frac{5}{3}, x_{q}>1\right\}, \\
& B_{h}=\left\{\widehat{x}=\left(x_{1}, \ldots, x_{q}\right) \in \widehat{B} ;\|\overrightarrow{e \widehat{x}}\|=\frac{5}{3}, x_{q}>1\right\},
\end{aligned}
$$


where $e$ denotes the centre of $\widehat{B}$. By choosing $\varphi$ sufficiently small, we may assume that $B_{h} \cap C_{\varphi} \neq \phi$ and $B_{l} \cap C_{\varphi} \neq \phi$.

Let $\gamma$ be a piecewise smooth path in $\widehat{M}^{*}$ from $\widehat{y}$ to a point in $\widehat{l}^{*}$. Since $\widehat{y}$ is not contained in $\widehat{B}$, we have the following possibilities:

1) $\gamma$ intersects both $B_{l}$ and $B_{h}$, or

2) $\gamma$ intersects $C_{\varphi}$.

If we have case 2), then by Sublemma 5.12 we have $\operatorname{length}_{\widehat{d}}(\gamma)>\varphi$.

Now we assume that $\gamma$ does not intersect $C_{\varphi}$, so we have case 1 ). Since $\gamma$ does not intersect $C_{\varphi}$, we may assume $\gamma\left[t_{0}, t_{1}\right]=\gamma[0,1] \cap B_{m}$. Recall that we have $r(\widehat{y}) \leq\|y\|$ for any $\widehat{y} \in \widehat{B}$; then

$$
\begin{aligned}
\operatorname{length}_{\widehat{d}}(\gamma) & >\int_{t_{0}}^{t_{1}}\left\|\frac{d \gamma}{d t}(t)\right\|_{\widehat{g}} d t \\
& \geq \int_{t_{0}}^{t_{1}}\left\|\frac{d\|\gamma\|}{d t}(t)\right\|_{\widehat{g}} d t \\
& \geq \int_{t_{0}}^{t_{1}} \frac{3}{5}\left\|\frac{d\|\gamma\|}{d t}(t)\right\| d t \\
& \geq \frac{1}{5}
\end{aligned}
$$

If we put $\epsilon_{0}=\min \left\{\frac{1}{5}, \varphi\right\}$, then we have $\widehat{d}\left(\widehat{y}, \widehat{l}^{*}\right)>\epsilon_{0}$.

Now we return to prove Proposition 5.11. Notice that $\widetilde{L}_{t}=p^{-1} \widehat{l}^{*}(t)$ is a single leaf of $\widetilde{\mathcal{F}}$. By Lemma 5.5 we have a compact foliated manifold $M_{R}=M \backslash \operatorname{Int} U$ which contains $\pi\left(p^{-1}\left(\widehat{l}^{*}\right)\right)$, where Int $U$ denotes the interior of $U$. Since $M_{R}$ is compact, we can choose an increasing sequence $\left\{t_{i}\right\}$ of positive real numbers such that if we choose points $c_{i}$ of $\widetilde{L}_{t_{i}}$ appropriately, then $\left\{\pi\left(c_{i}\right)\right\}$ converges to a point $c$ in $M_{R}$. Let $L_{c}$ be the leaf of $\mathcal{F}$ which contains $c$, and $\widetilde{L}_{c}$ a lift of $L_{c}$. Now we assume $d\left(\pi\left(c_{1}\right), c\right)$ is small enough; then by Sublemma 5.13 we can find a point $\widehat{b} \in \widehat{B}$ which satisfies $p\left(\widetilde{L}_{c}\right) \in \pi_{1}(M) \widehat{b}$. So we may assume that $\widehat{b}=p\left(\widetilde{L}_{c}\right) \in \widehat{B}$.

Then there is a sequence $\left\{\gamma_{i}\right\}$ of $\pi_{1}(M)$ such that $\widehat{d}\left(\widehat{b}, \gamma_{i}^{-1}\left(\widehat{l}^{*}\left(t_{i}\right)\right)\right)$ converges to zero. Since $\pi_{1}(M)$ acts on $\widehat{M}^{*}$ as an isometry, we see that $\widehat{d}\left(\gamma_{i}(\widehat{b}), \widehat{l}^{*}\left(t_{i}\right)\right)$ converges to zero. Then we may assume by virtue of Sublemma 5.13 that $\gamma_{i}(\widehat{b}) \in \widehat{B}$.

Now we pass to the model $B$, and take elements $f_{i}=\phi\left(\gamma_{i}\right)$ of $\operatorname{Sim}\left(\mathbf{R}^{q}\right)$ and $b=D\left(L_{c}\right) \in B$. Then by passing to a subsequence if necessary, we have

1) $f_{i}(b) \in B$.

2) $\theta\left(f_{i}(b)\right)$ converges to 0 .

3) $f_{i}(b)$ converges to $0 \in \mathbf{R}^{q}$.

For an element $f$ of $\operatorname{Sim}\left(\mathbf{R}^{q}\right)$, we write $f(x)=r A x+v$, where $r>0, A \in \operatorname{SO}(q)$, and $v \in \mathbf{R}^{q}$. Then we define $|f|=r$, and $P(f)=A$. Now we may assume

4) $P\left(f_{i}\right)$ converges to an element $P_{0}$ of $\mathrm{SO}(q)$.

Noticing that

$$
\left|f_{i}\right|=\frac{r\left(f_{i}(b)\right)}{r(\widehat{b})} \leq \frac{\left\|f_{i}(b)\right\|}{r(\widehat{b})},
$$

we have

5) $\left|f_{i}\right|$ converges to 0. 
Furthermore we may assume, by taking $i$ and $j$ sufficiently large, that $(j \gg i \gg 1)$

6) $P\left(f_{i} f_{j}^{-1}\right)$ is sufficiently near the identity element id of $\mathrm{SO}(q)$.

7) $\left|f_{j} f_{i}^{-1}\right|=\left|f_{j}\right| /\left|f_{i}\right|$ is sufficiently near 0 .

Now by 2$), \overrightarrow{0 f_{j}(b)}$ is almost parallel to $e$, and hence almost perpendicular to $\partial B$. Then by 6 ) and the fact that $f_{i} f_{j}^{-1}$ is a Euclidean similarity transformation, we see that

8) $\overrightarrow{f_{i} f_{j}^{-1}(0) f_{i}(b)}$ is almost parallel to $e$ and almost perpendicular to $f_{i} f_{j}^{-1}(\partial B)$. Notice that since $f_{i} f_{j}^{-1}\left(f_{j}(b)\right) \in B, \gamma_{i} \gamma_{j}^{-1} B^{*} \cap B^{*}$ is nonempty. Moreover, by the similarity, $f_{i} f_{j}^{-1} B \cap B$ is connected, hence $\gamma_{i} \gamma_{j}^{-1} B^{*} \cap B^{*}$ is a copy of $f_{i} f_{j}^{-1} B \cap B$. So $\gamma_{i} \gamma_{j}^{-1} B^{*} \cup B^{*}$ is a copy of $f_{i} f_{j}^{-1} B \cup B$. Then the completeness of $l$ shows that

9) $f_{i} f_{j}^{-1}(0) \notin B$, and

10) $f_{i} f_{j}^{-1}(B) \not \supset 0$.

We define $\delta_{j}$ to be the Euclidean distance between $f_{j}(b)$ and $\partial B$. Then condition 2) implies that $\delta_{j} /\left\|f_{j}(b)\right\|$ converges to 1 . Notice that the open ball $D_{\delta_{j}}\left(f_{j}(b)\right)$ centred at $f_{j}(b)$, of radius $\delta_{j}$, is contained in $B$.

Let $\epsilon$ be a positive real number less than 1 . Then we may assume that we have $\delta_{i} /\left\|f_{i}(b)\right\|>1-\epsilon / 2$, and $\left\|f_{i}(b)\right\|<\epsilon$.

Now we claim that $\left\|f_{i} f_{j}^{-1}(0)\right\|<3 \epsilon$ holds for any integers $i$ and $j$. Suppose the contrary; then we can find integers $i$ and $j$ such that $\left\|f_{i} f_{j}^{-1}(0)\right\| \geq 3 \epsilon$. Then we have

$$
\begin{aligned}
\left|f_{i} f_{j}^{-1}\right| & =\frac{\left\|\overrightarrow{f_{i} f_{j}^{-1}(0) f_{i}(b)}\right\|}{\left\|\overrightarrow{0 f_{j}(b)}\right\|} \frac{\left\|\overrightarrow{0 f_{i} f_{j}^{-1}(0)}\right\|-\left\|\overrightarrow{0 f_{i}(b)}\right\|}{\left\|\overrightarrow{0 f_{j}(b)}\right\|} \\
> & \frac{3 \epsilon-\epsilon}{\left\|\overrightarrow{0 f_{j}(b)}\right\|}=\frac{2 \epsilon}{\left\|f_{j}(b)\right\|} .
\end{aligned}
$$

On the other hand, since $D_{\delta_{j}}\left(f_{j}(b)\right)$ is contained in $B$, the ball

$$
f_{i} f_{j}^{-1}\left(D_{\delta_{j}}\left(f_{j}(b)\right)\right)=D_{\left|f_{i} f_{j}^{-1}\right| \delta_{j}}\left(f_{i}(b)\right)
$$

is contained in $f_{i} f_{j}^{-1}(B)$.

Now we have, however,

$$
\left|f_{i} f_{j}^{-1}\right| \delta_{j}>2 \epsilon \frac{\delta_{j}}{\left\|f_{j}(b)\right\|}>2 \epsilon\left(1-\frac{\epsilon}{2}\right)=2 \epsilon-\epsilon^{2}>\epsilon .
$$

This shows that the origin is contained in $D_{\left|f_{i} f_{j}^{-1}\right| \delta_{j}}\left(f_{i}(b)\right)$, because $\left\|f_{i}(b)\right\|<\epsilon$. Hence $f_{i} f_{j}^{-1}(B)$ contains the origin. This contradicts property 10). Thus the claim is proved.

Consequently we may assume $d\left(0, f_{i} f_{j}^{-1}(0)\right)$ is very small. Now 7$\left.\left.), 8\right), 9\right)$, and 10) show that $f_{i} f_{j}^{-1}(\partial B)$ tends to the hyperplane $\left\{x \in \mathbf{R}^{q} ; x_{q}=0\right\}$. This shows that for any $x \in\left\{x_{q}>0\right\}$, we can find integers $j \gg i \gg 1$ such that $f_{i} f_{j}^{-1} B \ni x$. Thus the union $\bigcup_{j \gg i \gg 1} f_{i} f_{j}^{-1} B \cup B$ contains a half space $H$ which contains $B$. Since $f_{i} f_{j}^{-1} B \cup B$ has a copy containing $\widehat{a}, H$ also has a copy $\widehat{H}$ containing $\widehat{B}$. 
Now we show the uniqueness. Let $\widehat{H}$ be a copy of a half space containing $\widehat{B}$. It is clear that $H=\Delta(\widehat{H})$ is tangent to $\Delta(\widehat{B})=D_{r(a)}(a)$ and the radius to the point of the tangency is the developing image of a short complete line. That is, there is a unique short complete line in $\widehat{M}^{*}$ which is contained in $\widehat{B}$. This shows the uniqueness of $\widehat{H}$.

Definition 5.14. We denote by $\widehat{H}(\widehat{a})$ the unique copy of the half space as in Proposition 5.11. We put $H(\widehat{a})=\Delta(\widehat{H}(\widehat{a}))$. Finally we denote by $\tau(\widehat{a})$ the point of tangency of $H(\widehat{a})$ and $D_{r(a)}(a)$.

Hereafter we use the same notations and conventions as in the proof of Proposition 5.11. Notice then that $\tau(\widehat{a})=0$.

Since $\Delta$ is a local homeomorphism, it is injective on the closure $\overline{\widehat{H}(\widehat{a})}$ of $\widehat{H}(\widehat{a})$ in $\widehat{M}^{*}$, and $\Delta(\partial \widehat{H}(\widehat{a}))$ is an open set of $\partial H(\widehat{a})$, the frontier of $H(\widehat{a})$ in $\mathbf{R}^{q}$.

Definition 5.15. We define $L(\widehat{a})=\partial H(\widehat{a}) \backslash \Delta(\partial \widehat{H}(\widehat{a}))$.

Here we immediately see that $x \in L(\widehat{a})$ if and only if there is a short complete line $\widehat{l}^{*}$ such that $\widehat{l}^{*}(0)=\widehat{a}$ and $\lim _{t \rightarrow \infty} l(t)=x$. For any point $y$ in $\partial H(\widehat{a}) \backslash L(\widehat{a})$, we denote by $\widehat{y}$ the unique point in $\partial \widehat{H}(\widehat{a})$ such that $\Delta(\widehat{y})=y$.

Lemma 5.16. For any point $x$ in $\overline{H(\widehat{a})} \backslash L(\widehat{a}), \partial H(\widehat{x})$ passes through $\tau(\widehat{a})$, where $\widehat{x}$ is the lift of $x$ in $\widehat{\widehat{H}(\widehat{a})}$.

Proof. First we choose a point $\widehat{y}$ near $\widehat{x}$ contained in $\widehat{H}(\widehat{a})$. Then $\widehat{y} \in \widehat{H}(\widehat{a}) \cap \widehat{H}(\widehat{x})$. So $H(\widehat{a}) \cup H(\widehat{x})$ has a copy in $\widehat{M}^{*}$. Hence $\tau(\widehat{a}) \notin H(\widehat{x})$.

Now we suppose that $\tau(\widehat{a}) \notin \partial H(\widehat{x})$. We consider the transformation $f_{j} f_{i}^{-1}=$ $\left(f_{i} f_{j}^{-1}\right)^{-1}$. Recall that $\left|f_{j} f_{i}^{-1}\right|$ is so small that $f_{j} f_{i}^{-1}$ acts as a strong contraction; then since the points $f_{i}(b), f_{j}(b)$ and $\tau(\widehat{a})$ are sufficiently near, it follows by the contraction principle that $f_{j} f_{i}^{-1}$ has a fixed point near $\tau(\widehat{a})$.

On the other hand, $P\left(f_{j} f_{i}^{-1}\right)$ is sufficiently near the identity. Consequently $f_{j} f_{i}^{-1} \partial H(\widehat{x})$ is almost parallel to $\partial H(\widehat{x})$ and nearer to $\tau(\widehat{a})$.

Now we may assume that $f_{j} f_{i}^{-1}(\Delta(\widehat{y})) \in H(\widehat{a})$, since $P\left(f_{j} f_{i}^{-1}\right)$ is near the identity. Hence we see that $\widehat{H}(\widehat{a}) \cap \gamma_{j} \gamma_{i}^{-1} \widehat{H}(\widehat{x}) \neq \phi$. Therefore $H(\widehat{a}) \cup H(\widehat{x}) \cup$ $f_{j} f_{i}^{-1} H(\widehat{x})$ has a copy in $\widehat{M}$. This contradicts the fact that $\tau(\widehat{x}) \in L(\widehat{x})$.

Lemma 5.17. $L(\widehat{a})$ is an Affine subspace of $\mathbf{R}^{q}$.

Proof. Let $x, y$ in $L(\widehat{a})$. We can write $x=\tau(\widehat{c})$ for some $\widehat{c} \in H(\widehat{a})$, and $y=\tau(\widehat{d})$. Notice that $L(\widehat{a})=L(\widehat{c})=L(\widehat{d})$. Suppose that there is a point $b$ on the line passing through $x$ and $y$ and which does not belong to $L(\widehat{a})$. Since $b$ is in $\partial H(\widehat{a})$, we can apply Lemma 5.16 to $b$ and see that $\partial H(\widehat{b})$ passes through $\tau(\widehat{c})=x$ and $\tau(\widehat{d})=y$. So $\partial H(\widehat{b})$ must pass through $b$. This is absurd.

Lemma 5.18. The correspondence $\widehat{a} \mapsto L(\widehat{a})$ is locally constant.

Proof. For any point $\widehat{b}$ of $\widehat{H}(\widehat{a})$, Lemma 5.16 shows that $\partial H(\widehat{b})$ passes through $\tau(\widehat{a})$, and $\tau(\widehat{a}) \in L(\widehat{b})$. Since $L(\widehat{b}) \cap H(\widehat{a})=\phi$ and $\tau(\widehat{a}) \in L(\widehat{b}) \cap \partial H(\widehat{a})$, we have $L(\widehat{b}) \subset \partial H(\widehat{a}) \cap \partial H(\widehat{b})$. Similarly we have $L(\widehat{a}) \subset \partial H(\widehat{a}) \cap \partial H(\widehat{b})$. 
Notice that $H(\widehat{a}) \cup H(\widehat{b})$ has a copy since $\widehat{b} \in \widehat{H}(\widehat{a}) \cap \widehat{H}(\widehat{b})$. Now let $x \in L(\widehat{a}) \backslash L(\widehat{b})$; then the fact that $x \in L(\widehat{a})$ shows that $x \notin \widehat{M}^{*}$. On the other hand, the fact that $x \notin L(\widehat{b})$ shows that $x \in \widehat{M}^{*}$.

This is impossible, and hence $L(\widehat{a}) \subset L(\widehat{b})$. The same argument shows that $L(\widehat{a}) \supset L(\widehat{b})$. Thus we obtain the result.

Since $\widehat{M}$ is connected, $L(\widehat{a})$ is independent of the choice of $\widehat{a} \in \widehat{M}$. So we write $L_{\infty}=L(\widehat{a})$. We may assume that $L_{\infty}$ is a vector subspace of $\mathbf{R}^{q}$.

Now we show that the image of $\Delta$ does not contain the point $\infty$.

Proposition 5.19. The mapping $\Delta$ is a covering map onto a component of $\mathbf{R}^{q} \backslash L_{\infty}$.

Proof. It is clear that the image of $\widehat{M}$ by $\Delta$ is contained in $\mathbf{R}^{q} \backslash L_{\infty} \cup\{\infty\}$. The above lemmas show that $\left.\Delta\right|_{\widehat{M}^{*}}: \widehat{M}^{*} \rightarrow \mathbf{R}^{q} \backslash L_{\infty}$ is a covering map. So it suffices to show that $\Delta^{-1}(\infty)=\phi$. This is clear in the case where $\operatorname{dim}_{\mathbf{R}} L_{\infty}>0$, because $\infty \in \overline{L_{\infty}}$.

Now suppose that $\operatorname{dim}_{\mathbf{R}} L_{\infty}=0$, i.e. $L_{\infty}=\{0\}$, and $\Delta^{-1}(\infty) \neq \phi$. If $q>2$, then $\mathbf{R}^{q} \backslash\{0\}$ is simply connected and hence $\left.\Delta\right|_{\widehat{M}^{*}}$ is a homeomorphism. Thus $\widehat{M}$ is homeomorphic to $\widehat{\mathbf{R}^{q}} \backslash\{0\}$. This contradicts our assumption.

We assume $q=2$; then $\widehat{M}^{*}$ is a covering of $\mathbf{R}^{2} \backslash\{0\}$ with covering degree $n$. If $n=0$, then the same argument shows that $\widehat{M} \cong \mathbf{R}^{2}$, which is a contradiction. If we have $n \neq 0$, then it is clear that $\Delta$ cannot be a local homeomorphism near the point $\infty$. This is again a contradiction.

Proof of Theorem C. Let $\operatorname{dim}_{\mathbf{R}} L_{\infty}=q_{0}$ and consider $\mathbf{R}^{q}=\mathbf{R}^{q_{0}} \times \mathbf{R}^{q_{1}}$, where $q_{1}=q-q_{0}$. When going down to $\mathbf{R}^{q}, \bar{g}$ is given by

$$
\bar{g}_{x}=\frac{1}{\left\|x_{2}\right\|^{2}} g_{0 x}, \text { where } x=\left(x_{1}, x_{2}\right) \in \mathbf{R}^{q_{0}} \times \mathbf{R}^{q_{1}} .
$$

This proves the theorem.

\section{REFERENCES}

1. B. N. Apanasov, Kobayashi conformal metric on manifolds, Chern-Simons and $\eta$-invariants, International J. Math. 2 (1991), 361-382. MR 92f:58027

2. T. Asuke, Classification of Riemannian flows with transverse similarity structures, preprint.

3. R. A. Blumenthal, Stability theorems for conformal foliations, Proc. Amer. Math. Soc. 91 (1984), 485-491. MR 86b:57013

4. Y. Carrière, Flots riemanniens, Structures transverses des feuilletages, Astérisque, vol. 116, 1984, pp. 31-52. MR 86m:58125a

5. D. Fried, Closed similarity manifolds, Comment. Math. Helvetici 55 (1980), 576-582. MR 83e: 53049

6. E. Ghys, Flots transversalement affines et tissus feuilletes, Mem. Soc. Math. France (N.S.) 46 (1991), 123-150. MR 92i:57026

7. J. Heitsch and S. Hurder, Secondary classes, Weil measures and the geometry of foliations, J. Diff. Geom. 20 (1984), 291-309. MR 86i:57032

8. R. S. Kulkarni and U. Pinkall, A canonical metric for Möbius structures and its applications, Math. Z. 216 (1994), 89-129. MR 95b:53017

9. S. Matsumoto, Foundations of flat conformal structure, Aspects of Low Dimensional Manifolds, Advanced Studies in Pure Mathematics 20, 1992, pp. 167-261. MR 93m:57014 
10. P. Molino, Riemannian Foliations, Progress in Mathematics, vol.73, Birkhäuser, 1988. MR 89b:53054

11. T. Nishimori, A note on the classification of nonsingular flows with transverse similarity structures, Hokkaido Math. J. 21 (1992), 381-393. MR 93h:58119

12. J. Plante, Foliations with measure preserving holonomy, Ann. Math. 102 (1975), 327-361. MR 52:11947

13. I. Vaisman, Conformal foliations, Kodai Math. J. 2 (1979), 26-37. MR 80g:57038

3-8-1 Komaba, Meguro-Ku, Tokyo 153, Japan

E-mail address: asuke@ms.u-tokyo.ac.jp 